\title{
Intracellular Localization of the HLA Class II Gene Products in Transfected Mouse $\mathrm{L}$ Cells
}

\author{
Takako Nakatsuji ${ }^{1), *}$ and Akira Akatsuka ${ }^{2)}$ \\ 1)Department of Transplantation and ${ }^{2)}$ Division of Central Electron-microscope, Tokai University School of \\ Medicine, Isehara, Japan
}

Key words: HLA class II antigen/morphology/transfectants

\begin{abstract}
To investigate the impaired cell surface expression of human major histocompatibility antigen (HLA) in transfected L cells, we examined their intracellular localization by immunocytochemistry. HLA class II molecules produced in transfected $\mathrm{L}$ cells were mainly detected in the intracellular vesicles and in the nuclear envelope as granular precipitates. The results suggest that the intracellular transport of the newly synthesized molecules in transfected $L$ cells is impaired at some point along the pathway from the rough endoplasmic reticulum (RER) to the medial-, trans-Golgi apparatus.
\end{abstract}

The HLA class II antigens are polymorphic cell surface glycoproteins. Expression of class II molecules is restricted to certain cells for antigen presentation. These molecules bind immunogenic peptides, and present them to T lymphocytes (8). Such peptides are normally derived from protein antigens internalized and proteolytically degraded in antigen-presenting cells. The normally biosynthesized $\alpha$ and $\beta$ chains of class II molecules associate with the invariant (Ii) chain in the RER. These intracellular class II molecules are transported from the RER to Golgi apparatus, and the Ii chain is proteolytically removed in an endosomal compartment before cell surface expression (14). The immunogenic peptides can bind to class II molecules in the endosomal compartment only after proteolytic cleavages of the Ii chain from the $\alpha$ - $\beta$-Ii complex $(14,3,16)$.

To elucidate biological functions of each single HLA class II antigen molecule, we transfected various cells with the HLA class II gene (12). However, expression of the HLA class II antigen in the transfected L cells was very weak (13). In this study, we used ultrastructural immunocytochemistry to examine intracellular localization of the HLA class II antigen expressed in transfected $\mathrm{L}$ cells and normal human B-lymphocytes.

\section{MATERIALS AND METHODS}

Transfected $L$ cells. Cosmid clones obtained from B cell

\footnotetext{
* To whom correspondence should be addressed.

Abbreviations: HLA, human leucocyte antigens, human major histocompatibility antigens; RER, rough endoplasmic reticulum; Ii, invariant chain; FCS, fetal calf serum; PBS, phosphate-buffered saline; DMEM, Dulbecco's modified Eagle's medium.
}

line (AKIBA, PGF) gene libraries were cotransfected with pSV2neo gene into mouse $L$ cells by means of the modified calcium phosphate method (2). The cosmid clones pAKR 4643 (DRB1) or pAKR4608-2 (DRB5, previously named DR $\beta I I I)$ were cotransfected with pAKR5214-3 (DR $\alpha$ ). The pPR503 $(\mathrm{DR} \alpha)$ and pPR241 (DRB5) were cotransfected as another pair (Table I) (5). $5 \times 10^{5}$ cells were cultured with $20-30 \mu \mathrm{g}$ of DNA in $2 \times$ BES buffer saline ( $50 \mathrm{mM} \mathrm{N}$-, N-Bis(2-hydroxyethyl)-2-aminoethanesulfonic acid, $280 \mathrm{mM} \mathrm{NaCl}, 1.5 \mathrm{mM}$ $\mathrm{Na}_{2} \mathrm{HPO}_{4}$ ) supplemented with $0.25 \mathrm{M} \mathrm{CaCl}_{2}$. After culturing for $15-24 \mathrm{hr}$ under the condition of $35^{\circ} \mathrm{C}, 2-4 \% \mathrm{CO}_{2}$, the medium was changed to DMEM supplemented with $10 \%$ FCS. The transfected cells were selected and maintained using 400 $\mu \mathrm{g} / \mathrm{ml}$ Geneticin (GIBCO). Transfected cells positive for the class II antigen were sorted with the monoclonal antibody L243, and cloned.

Monoclonal antibodies. L243 (anti HLA-DR) (6), Leu10 (anti HLA-DQ) (1) and B7/21 (anti HLA-DP) (19) were purchased commercially (Becton Dickinson). DA6.231 and DA6.147 ascites were gifts from Dr. Keith Guy (4).

Flow microfluorimetric analysis. Antigen expression of transfected L cells was analyzed by FACStar (Becton Dickinson). Washed $1 \times 10^{6}$ cells were incubated for $30 \mathrm{~min}$ with $50 \mu \mathrm{l}$ of HLA class II monoclonal antibody at a concentration optimal for staining the positive control, a B cell line. After washing with phosphate-buffered saline (PBS), cells were incubated for $15 \mathrm{~min}$ with $50 \mu \mathrm{l}$ of fluorescein isothiocyanate (FITC)-conjugated $F(a b)_{2}$ goat anti-mouse immunoglobulin (Cappel) at a dilution of $1: 15$. Cell surface staining was analyzed by means of a fluorescence-activated cell sorter (FACStar).

Immunoperoxidase staining for light and electron microscopy. The transfectants cultured on Lab-Tek (Miles Scien- 
Table I. Genomic HLA genes for tRansfectants.

\begin{tabular}{llc}
\hline cell lines & \multicolumn{1}{c}{ DNA clones } & HLA gene names \\
\cline { 1 - 2 } LARB1 & pAKR5214-3 + pAKR4643 & DRA + DRB1 \\
LARB5 & pAKR5214-3 + pAKR4608-2 & DRA + DRB5 \\
LPRB5 & pPR503 + pPR241 & DRA + DRB5 \\
\hline
\end{tabular}

tific) were fixed with $4 \%$ paraformaldehyde for $3 \mathrm{hr}$ at $4{ }^{\circ} \mathrm{C}$. The $\mathrm{B}$ cell line cells in a culture flask were washed out and transferred to a microplate using a Cytospin. After freezing for $1 \mathrm{hr}$, the cells were thawed at $37^{\circ} \mathrm{C}$ for $30 \mathrm{~min}$ to facilitate efficient penetration of antibodies. After three washes (the first with PBS, the second with 5\% sucrose in PBS and the third with $10 \%$ sucrose in PBS), the endogenous peroxidase was blocked. These samples were incubated overnight at $4^{\circ} \mathrm{C}$ with a cocktail of HLA class II monoclonal antibodies, including L243, DA6.231, DA6.147, Leu10 and B7/21, at concentrations predetermined with the positive control B cell line AKIBA. A cocktail of antibodies was used to detect various biosynthetic products of the class II genes. After washing with cold PBS, cells were incubated with horseradish peroxidase (HRP)-labeled sheep anti-mouse Ig F(ab') ${ }_{2}$ for $3 \mathrm{hr}$. After fixation with $1 \%$ glutaraldehyde for $20 \mathrm{~min}$, samples were incubated with DAB (3,3'-diaminobenzidine; SIGMA) for 30 min following $\mathrm{DAB}-\mathrm{H}_{2} \mathrm{O}_{2}$ treatment for $2 \mathrm{~min}$. For light micros- copy, cells were stained with methyl green. For electron microscopy, cells were postfixed for $1 \mathrm{hr}$ at room temperature with $2 \% \mathrm{OsO}_{4}$ (osmium tetroxide; SIGMA). They were dehydrated through graded ethanol series and embedded in Epon. The ultrastructural immunocytochemical procedures were carried out according to the method of Nakane and Pierce (11).

\section{RESULTS}

Cell surface expression of the HLA-DR antigen on transfected L cells, LARB1, LARB5 and LPRB5 was analyzed flowmicrofluorimetrically in comparison with a B cell line, AKIBA. Relative fluorescence intensity of the HLA-DR antigen expressed on the transfectants showed weakly positive surface expression in contrast to strong DR, DQ or DP expression on the AKIBA cell (Fig. 1). Therefore, cell surface expression of the newly synthesized HLA-DR antigen on the transfected mouse $\mathrm{L}$ cells was at a low level, less than $4 \%$ of the innate expression on the B cells. As shown in Fig. 1, the LARB1 cell showed slightly stronger binding with the monoclonal antibody L243 among three transfectants. LARB5 and LPRB5 cells showed a similar intensity (Fig. 1). Similar results were also obtained using the monoclonal antibodies DA6.231 and DA6.147 (data not shown).

In our preliminary studies, culture supernatants of

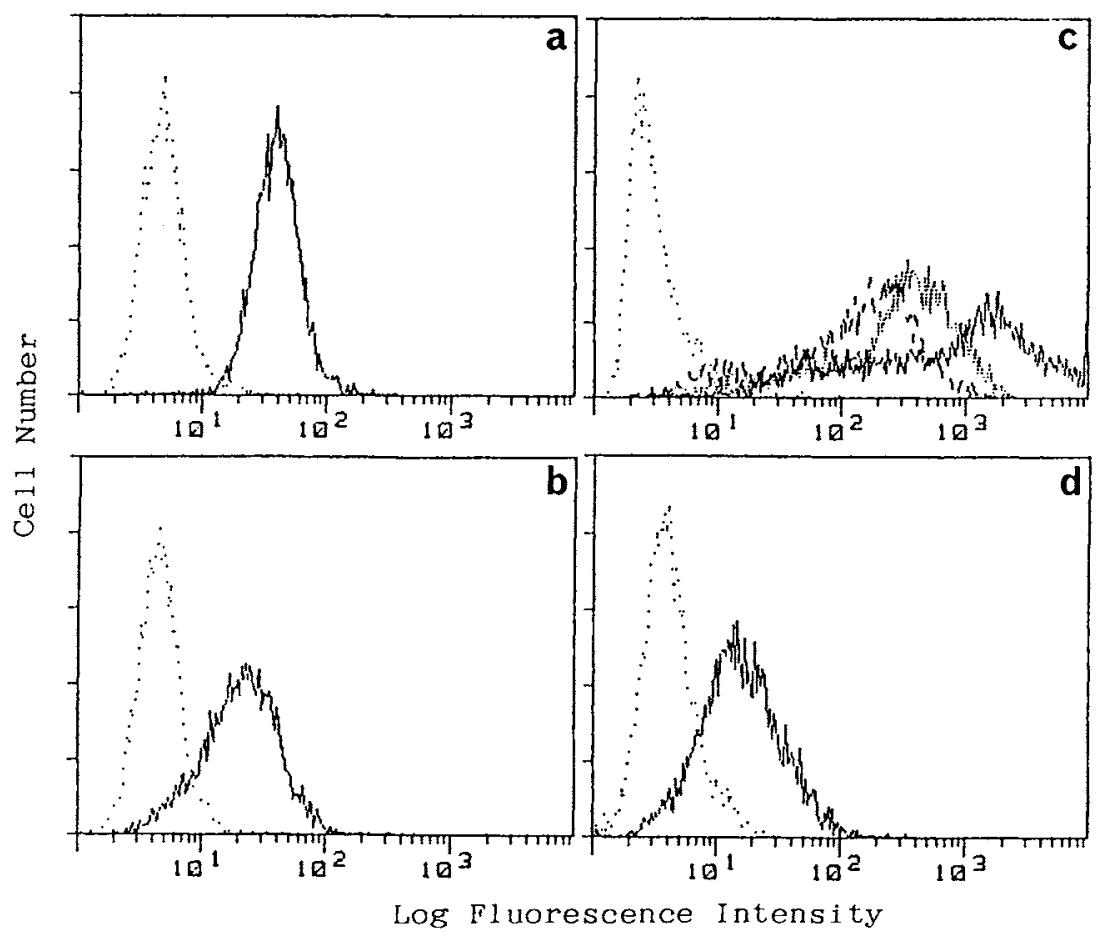

Fig. 1. Surface expression of HLA-DR antigens on transfectants LARB1 (a), LARB5 (b), LPRB5 (d), and B cell line AKIBA (c). The flow microfuorimetric analysis was carried out using monoclonal antibody L243 (- - Monoclonal antibodies, Leu $10(\ldots . . . .$.$) and B7/21 (---)$, were

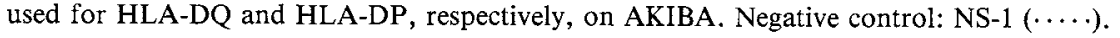


the transfectants and AKIBA cells were examined to detect any secreted or shed products of HLA-DR gene in the extracellular medium using a biosynthetically labeled immunoprecipitation method. Neither immunoblot analysis nor two-dimensional gel electrophoresis showed any precipitates in culture supernatants of the transfectants, although the same analyses demonstrated that AKIBA cells produced and released antigens into their media.

According to the light microscopical observation, immunoperoxidase staining of the transfectants and AKIBA cells showed remarkable differences (Fig. 2). As expected, a strong expression of the DR antigen was detected on the AKIBA cell surface (Fig. 2a). The transfected $L$ cells, however, did not have strong cell surface staining. Instead, staining for the HLA-DR molecules was mostly located in cytoplasmic granules. For example, immunoperoxidase staining products in LARB1 cells were almost always distributed intracellularly as shown in Fig. 2b. The LPRB5 cells also showed similar distribution (data not shown). Immunoperoxidase staining products in LARB5 cells were also distributed to the cytoplasm, but these cells showed a large variation of staining among cells (Fig. 2c); some cells had stronger than the LARB1 cells, while others had almost no staining (Fig. 2c).

Ultrastructural immunoperoxidase staining showed that the HLA-DR gene products of the transfectant LARB5 were distributed among intracellular vesicles and near the nuclear envelopes (Fig. 3a). These electron micrographs of the transfectant LARB5 show the representative staining pattern of the transfectants observed in this study. They show strong positive staining in the nuclear envelope and the intracellular vesicles (Fig. 3b). On the other hand, there was weakly positive staining on the cell surface membrane as shown in Fig. 3a. The LARB1 and LPRB5 also showed similar immunoperoxidase staining to the LARB5 cells: strong staining in intracellular locations but only weak staining on the cell surface (data not shown). Electron micrographs of the

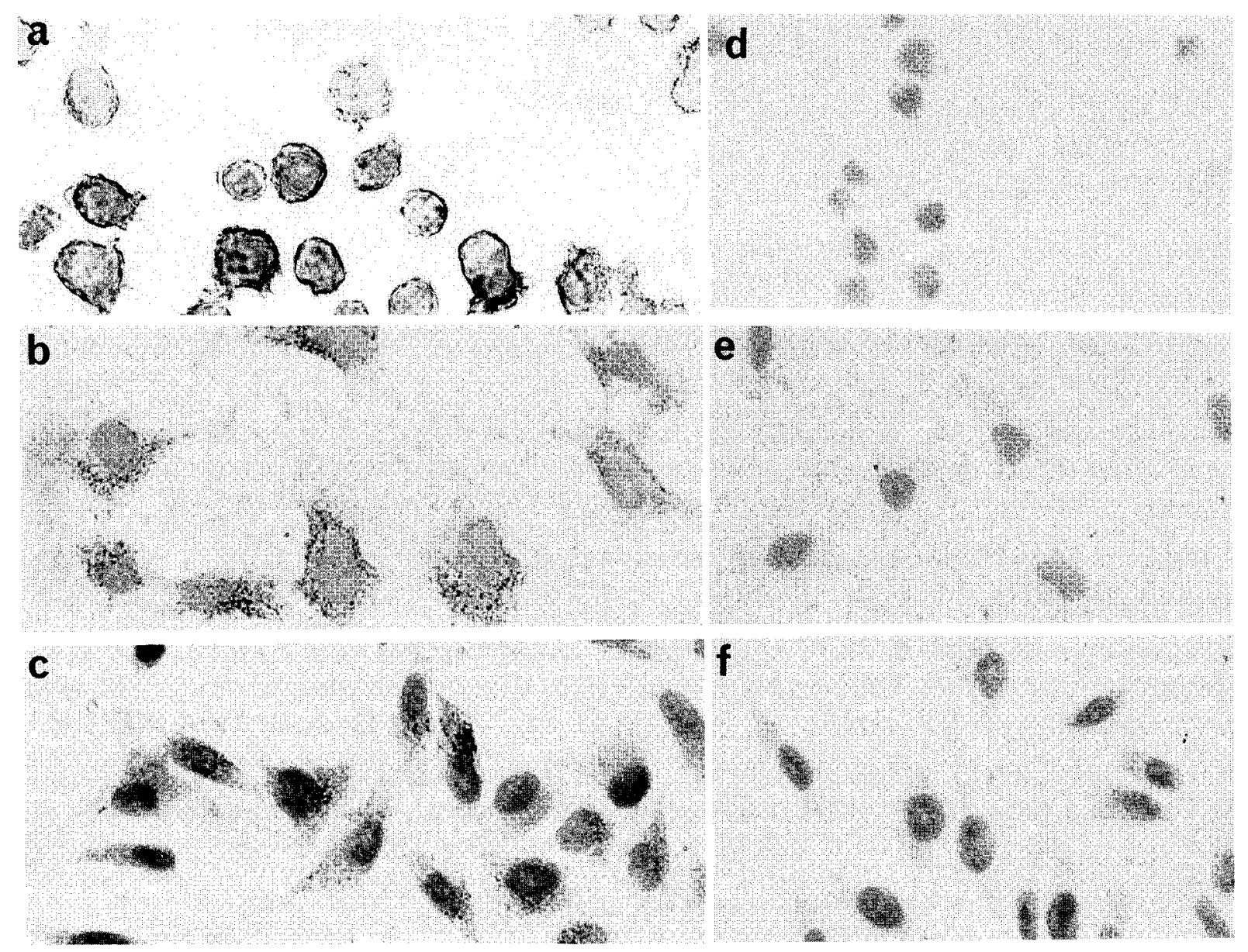

Fig. 2. Light micrographs of immunoperoxidase staining of AKIBA (a), LARB1 (b), and LARB5 (c). Negative controls (d, e, and f) of AKIBA, LARB1 and LARB5, respectively stained with the NS-1 ascites. Magnifications: $a, \times 750 ; \mathrm{b}$ and $\mathrm{c}, \times 600$. 
AKIBA cells showed clearly positive staining on the cell surface and periphery of the nuclear envelope (Figs. 3c and $3 \mathrm{~d})$.

In conclusion, these results indicated that the trans- fected HLA-DR gene products were distributed among cytoplasmic organelles, and thus clearly distinguishable from distribution of the innate HLA-DR gene products. The ultrastructural immunoperoxidase staining
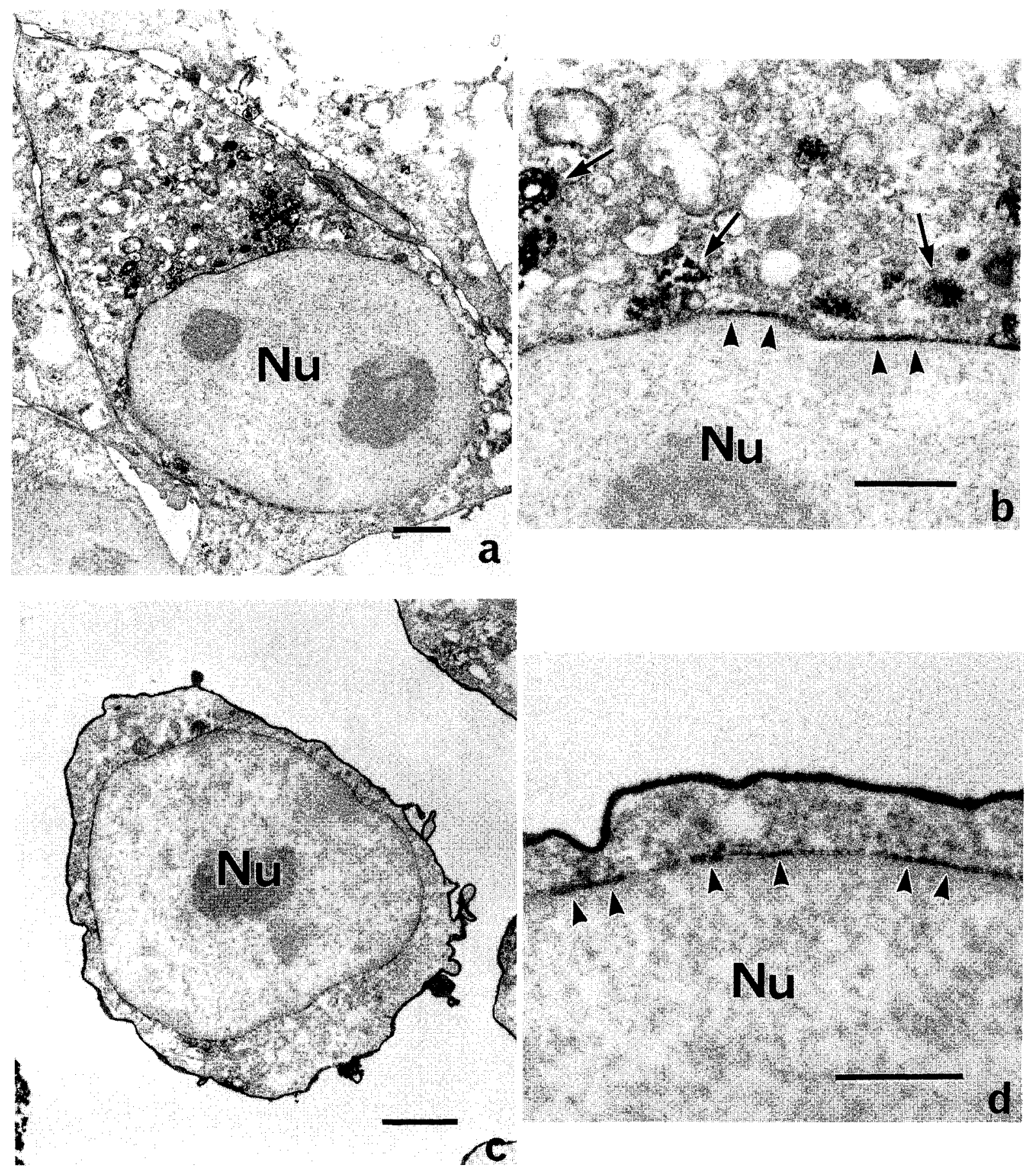

Fig. 3. Electron micrographs of the transfectant LARB5 (a) and B cell AKIBA (c). (b) and (d) are higher magnifications of (a) and (c), respectively. Immunoperoxidase staining of intracellular vesicles (arrows), nuclear envelope (arrowheads) and cell surface membrane was observed. Nu: nucleus. Scale bar indicates $2 \mu \mathrm{m}$ (lower magnification), or $1 \mu \mathrm{m}$ (higher magnification). 
products of the HLA-DR transfectants were mainly detected in the intracellular vesicles and the nuclear envelope, despite the fact that the mature HLA gene products, including HLA-DR, are cell surface glycoproteins in normal B cells.

\section{DISCUSSION}

In our previous studies, we noted that the expression of HLA class II antigens on transfected mouse L cells may be impaired (13). Also, we did not detect any secretion or shedding products of the HLA-DR gene. To investigate details of this impaired expression, intracellular distribution of the synthesized antigens was examined by ultrastructural immunocytochemistry. Surprisingly, the synthesized HLA class II antigen was accumulated in the intracellular vesicles.

Cell surface expression of the newly synthesized HLA-DR antigen on the transfected mouse $L$ cells was at a low level in the flow microfluorimetrical analysis. The immunoelectron-microscopy of these transfected cells showed results consistent with the low-level expression detected by the flow microfluorimetry. In our previous studies of immunoprecipitation (Nakatsuji et al., submitted for publication), the amounts of the HLADR molecules in the transfected mouse $\mathrm{L}$ cells were calculated as less than 7\% of the innate HLA-DR molecules in the AKIBA cells (13). Therefore, it is suggested that more than $90 \%$ of synthesized HLA-DR molecules in the transfected mouse L cells were transported to the intracellular vacuole system.

Immunoperoxidase staining intensity was rather variable among LARB5 cells. The LARB5 cells might be derived from more than one clone. It would be interesting if the variability could be observed even after cloning.

Our transfection was carried out without the li chain gene, as described in Materials and Methods section. Moreover, accumulation of the immunoperoxidase staining in the lysosomal organelles was similarly observed in both HLA-DQ and -DP gene transfection experiments (data not shown). The li chain might have a role in the cell surface expression. The major histocompatibility class II antigens, which are associated intracellularly with the Ii chain, are heterodimers composed of the $\alpha$-chain and $\beta$-chain on the cell surface. According to McMillan et al. (9), mouse major histocompatibility complex (H-2) class II genes transfected into B-lymphoma cells were highly expressed as compared with the gene transfected into fibroblasts. A study of HLA-DR gene transfer using a mouse fibroblast cell line, L cells, showed that their surface expression varied among clones (15). Only one clone expressed HLA-DR molecules at the same order of magnitude as the control $B$ cell line (15). However, many recent studies have revealed no significant influence of the presence of Ii chains on class II surface expression $(10,17,18)$. As dis- cussed by Guagliardi et al. (3), further studies are required to define the role of Ii chains: whether Ii chains directly influence peptide binding or account for the direction of class II molecules to endocytotic compartments.

Williams et al. (20) discussed requirements of proper conformation for intracellular transport and surface expression of mouse class I histocompatibility molecules. They studied a mutant $\mathrm{H}-2 \mathrm{~K}^{\mathrm{b}}$ molecule produced in a somatic cell variant and expressed on the cell surface at about $15 \%$ of the wild-type level. Eighty percent of the newly synthesized mutant molecules were not exported out of the RER, where they were slowly degraded. The remaining $20 \%$, however, were capable of reaching the plasma membrane at a near-normal rate. It is suggested that some structural aspects beyond a specific change in the primary sequence are involved in export of the molecules out of the RER. An intracellular degradation study indicated that the newly synthesized excess T-cell receptor chains in transfected fibroblasts are rapidly degraded in the endoplasmic reticulum system (7). It is suggested that unassembled or incomplete membrane protein complexes are disposed in a rapid degradative pathway different from the alternative lysosomal pathway (7). In our transfected cells, there was a possibility of unbalanced excess production of proteins because the HLA-DR $\alpha$ gene and $\beta$ gene were separately introduced into the cells.

In this study, we analyzed causes of the weakened surface expression of the HLA-DR gene transfected into L cells by use of immunolocalization methods at light and electron microscopical levels, and found accumulation of the products in the intracellular vesicles, suggesting that the transgene products require some potential to be transported to the cell surface.

Acknowledgments. This work was supported in part by Tokai Research Fund. The authors wish to acknowledge Dr. Norio Nakatsuji for critical reading of the manuscript and valuable discussion.

\section{REFERENCES}

1. BRODSKY, F.M. (1984). A matrix approach to human class II histocompatibility antigens: reaction of four monoclonal antibodies with products of nine haplotypes. Immunogenetics, 19: 179.

2. Chen, C., and Okayama, H. (1987). High-efficiency transformation of mammalian cells by plasmid DNA. Mol. cell. Biol., 7 : 2745.

3. Guagliardi, L.E., Koppelman, B., Blum, J.S., Mark, M.S., Crosswell, P., and Brodsky, F.M. (1990). Co-localization of molecules involved in antigen processing and presentation in an early endocytic compartment. Nature, 343: 133.

4. Guy, K., van Heyningen, V., Cohen, B.B., Deane, D.L., and STEEL, C.M. (1982). Differential expression and serologically distinct subpopulations of human la antigens detected with monoclonal antibodies to $\alpha$ and $\beta$ chains. Eur. J. Immunol., 12: 942. 
5. Kawai, J., Ando, A., Sato, T., Nakatsuji, T., Tsuit, K., and INoKo, H. (1989). Analysis of gene structure and antigen determinants of DR2 antigens using DR gene transfer into mouse $\mathrm{L}$ cells. J. Immunol., 142: 312.

6. LAMPSON, L.A. and LEVY, R. (1980). Two populations of Ialike molecules on a human B cell line. J. Immunol., 125: 293.

7. LippinCOTt-Schwartz, J., Bonifacino, J.S., YUan, L.C., and KLAUSNER, R.D. (1988). Degradation from the endoplasmic reticulum disposing of newly synthesized proteins. Cell, 54: 203.

8. LONG, E.O. (1989). Intracellular traffic and antigen processing. Immunology Today, 10: 232.

9. McMillan, M., White, J., Kappler, J., Marrack, P., Pierres, A., Pierres, M., and Hood, L. (1984). Gene transfer of H-2 class II genes: Antigen presentation by mouse fibroblast and hamster B cell lines. Cell, 36: 319.

10. Miller, J. and Germain, R.N. (1986). Efficient cell surface expression of class II MHC molecules in the absence of associated invariant chain. J. Exp. Med., 164: 1478.

11. Nakane, P.K. and Pierce, G.B.Jr. (1966). Enzyme-labelled antibodies: preparation and application for the localization of antigen. J. Histochem. Cytochem., 14: 929.

12. Nakatsuit, T., Inoko, H., Ando, A., Sato, T., Koide, Y., Tadakuma, T., Yoshida, T.O., and Tsuis, K. (1987). The role of transfected HLA-DQ genes in the mixed lymphocyte reaction-like condition. Immunogenetics, 25: 1.

13. Nakatsuji, T., Sato, T., Ito, K., Tsuchida, F., Ando, A., Sano, N., Matsuno, N., Inoko, H., KoIDe, Y., Yoshida, T.O., and Tsujı, K. (1989). Proliferative response by stimula- tion of DP-transfectants. In Immunology of HLA (B. Dupont ed.), Vol. 2. Immunogenetics and Histocompatibility. SpringerVerlag, p. 317.

14. NGuYen, Q.V. and Humphreys, R.E. (1989). Timecourse of Intracellular association, Processing and cleavages of Ii forms and class II major histocompatibility complex molecules. $\mathrm{J}$. Immunol., 264: 1631.

15. Rabourdin-Combe, C. and Mach, B. (1983). Expression of HLA-DR antigens at the surface of mouse L cells co-transfected with cloned human genes. Nature, 303: 670.

16. Roche, P.A. and Cresswell, P. (1990). Invariant chain association with HLA-DR molecules inhibits immunogenic peptide binding. Nature, 345: 615 .

17. Sekaly, R.P., Tonnelle, C., Strubin, M., Mach, B., and LONG, E.O. (1986). Cell surface expression of class II histocompatibility antigens occurs in the absence of the invariant chain. J. Exp. Med., 164: 1490.

18. Stockinger, B., Pessara, U., Lin, R.-H., Habicht, J., Grez, M., and KoCH, N. (1989). A role of Ia-associated invariant chains in antigen processing and presentation. Cell, 56: 683,

19. Watson, A.J., DeMars, R., Trowbridge, I.S., and Bach, F.H. (1983). Detection of novel human class II HLA antigen. Nature, 304: 348.

20. Williams, D.B., Borriello, F., ZefF, R.A., and Nathenson, S.G. (1988). Intracellular transport of class I histocompatibility molecules. Influence of protein folding on transport to the cell surface. J. Biol. Chem., 263: 4549.

(Received for publication, October 25, 1991 and in revised form, December 19, 1991) 